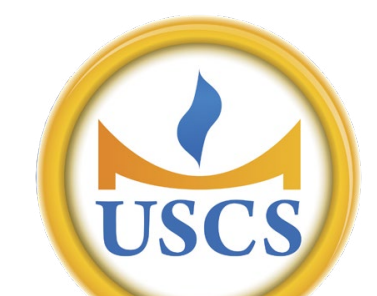

UNIVERSIDADE MUNICIPAL

DE SÃO CAETANO DO SUL

Publicação do Programa de Pós-graduação em Comunicação Social da Universidade Municipal de São Caetano do Sul

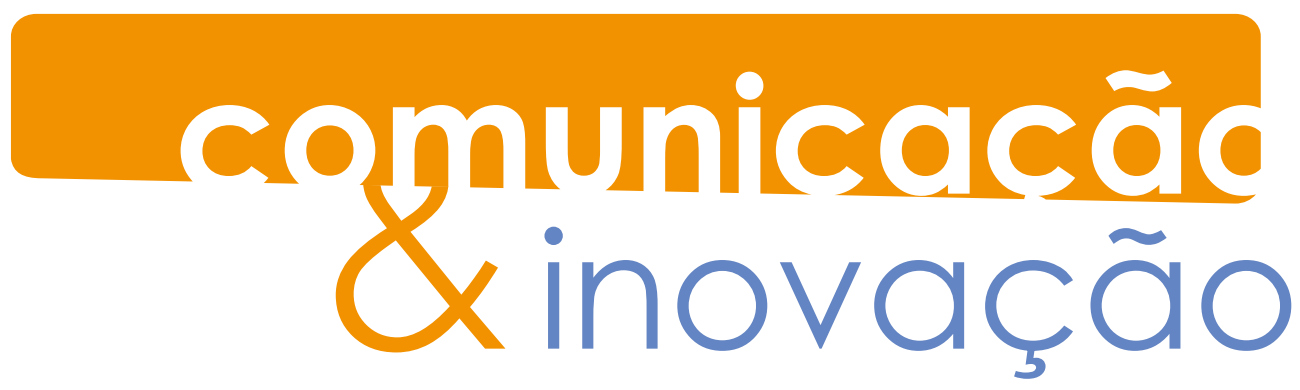

volume 18 • número 37 • maio-agosto 2017 


\section{communicacãd \\ Q inovação}

Integrada à RED - Iberoamericana de Revistas de Comunicação e Cultura

(www.revistadecomunication.org)

\section{Revistas integrantes da RED}

Actas y Comunicaciones del Instituto de Historia Antigua y Medieval

Anagramas

Anales de Historia Antigua, Medieval y Moderna

Anàlisi

Anuario de Investigaciones

Anuario ININCO

Anuario UNESCO/UMESP de Comunicación

Regional

Aportes de la Comunicación y la Cultura

Arandu

Arte Críticas

Boletín Científico Sapiens Research

C+I Comunicación e Información

Chasqui

Comunicação \& Artes

Comunicação \& Educação

Comunicação \& Inovação

Comunicação \& Sociedade

Comunicação Pro

COMUNICACIÓN U.P.B.

Comunicación y Hombre

Comunicación y Medios

Comunicación y Sociedad (México)

Comunicación y Sociedad (España)

Comunicación. Estudios Venezolanos de la Comunicación

Comunicar. Revista Científica Iberoamericana de

Comunicación y

Educación

Conexiones. Revista Iberoamericana de Comunicación

\author{
Constelaciones de la Comunicación \\ Contratexto \\ Convergencia. Revista de Ciencias Sociales \\ Criterio Libre \\ Cuaderno de H Ideas \\ Cuadernos de información \\ Dedica. Revista de Educação e Humanidades \\ Diálogos de la Comunicación \\ Disertaciones \\ Doxa.comunicación. Revista interdisciplinar de \\ comunicación y ciencias \\ sociales \\ Em Questão \\ Escribania \\ Espacios Públicos \\ Estudios de Periodismo y Relaciones Públicas \\ Estudios sobre las Culturas Contemporáneas \\ Exedra. Revista Científica \\ Extensión en Red \\ Figuraciones \\ Global Media Journal \\ Icono 14 \\ Imagofagia \\ In-mediaciones de la Comunicación \\ INSIGHTS \\ Interação \\ Interacción. Revista de Comunicación Educativa \\ Intercom: Revista Brasileira de Ciências da Co- \\ municação \\ Interlenguajes \\ Intersecciones \\ Isla Flotante
}

\section{DAdos INTERNACIONAIS de CATAlogaÇÃo Na PUbliCaÇão (CIP)}

Comunicação \& Inovação / [Publicação do] Curso de Comunicação Social da Universidade Municipal de São Caetano do Sul - v. 6, n. 11 jul/dez 2005 - São Caetano do Sul : Universidade IMES, 2005

Semestral

Resumo em inglês e português

Continuação da Revista IMES Comunicação, v. 1, n. 1-10, 2000-2005

ISSN 2178-0145

1. Comunicação - Periódico. I. Universidade Municipal de São Caetano do Sul 


\section{comunicacãd \\ \&inovação}

\section{Revistas integrantes da RED}

Izquierdas

La Trama de la Comunicación

Líbero

LIS. Letra. Imagen. Sonido. La Ciudad Mediatizada

Luciérnaga

Lumina

Más Poder Local

Mediaciones

Medios y Enteros

MiRatón

Newsletter

Oficios Terrestres

Ojos de Buey

Ojo de Buey. Revista digital de cine

Palabra Clave

Papeles de trabajo

Pedagogía Social. Revista Interuniversitaria

Perspectivas de la Comunicación

Pixel-Bit. Revista de Medios y Educación

Poliantea

Prisma Social

Punto Cero

Question

Quórum Académico

Razón y Palabra

Revista Científica Digital Pensamiento Comunicacional Latinoamericano
Revista de Educación Inclusiva

Revista de la Facultad de Derecho y Ciencias

Sociales

Revista Electrónica de Investigación y Docencia (REID)

Revista Electrónica Nodos de la Comunicación REVISTA FAMECOS: mídia, cultura e tecnologia

Revista Interamericana de Nuevas Tecnologías de la Información

Revista Internacional de Folkcomunicação - Revista Folkcom (on line)

Revista latina de Comunicación Social

Revista Mexicana de Comunicación

RIALAI

RIED. Revista Iberoamericana de Educación a

Distancia

Signo y Pensamiento

Sinergia

Tecnología y Comunicación Educativas

Telos. Cuadernos de Comunicación e Innovación

Temas de Comunicación

Temas y Problemas de Comunicación

Trama

Tram[p]as de la Comunicación y la Cultura

Ventana Indiscreta

Versión. Estudios de Comunicación y Política 


\title{
comunicacãc dinovação
}

\author{
volume 18 • número 37 \\ maio - agosto 2017
}

Fechamento desta edição: agosto 2017

\section{Reitor \\ Marcos Sidnei Bassi \\ Pró-Reitor Administrativo e Financeiro Paulo Sérgio Lopes Ruiz \\ Pró-Reitor de Graduação Leandro Campi Prearo \\ Pró-Reitor de Pós-Graduação e Pesquisa Maria do Carmo Romeiro}

\section{Gestor do Programa de Pós-Graduação Stricto Sensu em Comunicação João Batista de Freitas Cardoso}

\section{Editora \\ Regina Rosetti}

\section{Gestor de Comissão de Publicações Acadêmicas}

Marco Antonio Pinheiro da Silveira

\section{Jornalista Responsável}

Roberto Elísio dos Santos - MTb 15.637

\section{Comissão Editorial}

Dr. Arquimedes Pessoni

Dr. Roberto Elísio dos Santos

Dra. Regina Rossetti

\section{Conselho Consultivo}

Ángel Badillo Matos, USAL, Espanha

Ana Silvia Lopes Davi Médola, UNESP, Brasil

Dilma de Melo Silva, ECA/USP, Brasil

Eron Brum, Uniderp, Brasil

Fernando Andacht, University of

Ottawa, Canadá

Gilson Monteiro, UFAM, Brasil

Henrique Paiva de Magalhaes, UFPB, Brasil

Jean Louis Santorio, ENCPB, França

John Lent, School of Communications

and Theatre Temple University, EUA

José Antonio Moreiro Gonzalez,

Universidad Carlos III/Madri, Espanha

Juan Pablo Gonzalez, UC, Chile

Juremir Machado da Silva, PUC/RS, Brasil
Kleber Santos de Mendonça, UFF, Brasil

Laura Vazquez Hutnik, UBA, Argentina

Liana Gottlieb, Casper Líbero, Brasil

Maria Amélia Chagas Gaiarsa, UCSAL, Brasil

Maria Ataide Malcher, UFPA, Brasil

Maria das Graças Andrade Ataide de

Almeida, UFRPE, Brasil

Maria das Graças Targino, UFPI, Brasil

Maria Érica de Oliveira Lima, UFRN, Brasil

Maria Lucia Santaella Braga, PUC/SP, Brasil

Marcius Freire, Unicamp, Brasil

Marita Fornaro, Universidad de la

República, Uruguai

Nello Barile, IULM, Itália

Neusa Demartini Gomes, PUC/RS, Brasil

Nilda Aparecida Jacks, UFRGS, Brasil

Paulo Eduardo Ramos, UNIFESP, Brasil

Sandra Lucia Amaral de Assis Reimão,

ECA/USP, Brasil

Sergio Dayrell Porto, UNB, Brasil

Tanius Karam, UNAM, México

Winfried Maximilian Nöth, PUC/SP, Alemanha

\section{Coordenação Editorial}

Aline Maya e Luan Maitan | Tikinet

\section{Preparação e Revisão}

Andressa Picosque, Fernanda Corrêa, Gabriel Sousa, Hamilton Fernandes, Nicolas Leonezi, Stéphanie Roque | Tikinet

\section{Diagramação}

Natalia Bae e Robson Santos | Tikinet

\section{Comunicação \& Inovação}

Rua Santo Antônio, 50 - Centro

São Caetano do Sul - SP

Tel.: (11) 4239-3200 (ramal 3441)

E-mail: rrossetti@uscs.edu.br

Página na Web: http://seer.uscs.edu.br/index.

php/revista_comunicacao_inovacao

AUniversidade Municipal de São Caetano do Sul, em seus cadernos e revistas, respeita a liberdade intelectual dos autores, publica os originais que lhe são entregues, sem com isso concordar necessariamente com as opiniões expressas. 


\section{SUMARIO}

Artigos

Observatório da Comunicação Pública: Pesquisa, Crítica e CIDADANIA

Maria Helena Weber, Ana Javes Luz

O INTERESSE PÚBLICO E A LIBERDADE DE IMPRENSA COMO FUNDAMENTOS DA COMUNICAÇÃO JORNALÍSTICA DEMOCRÁTICA Maria Elisabete Antonioli, Cassio Cavalcante Andrade

Publicidade E INTEResse Público: Noções ARTICUladas EM INSTÂNCIA NORMaTIVA do Governo FEDERAL do BRASIL

Patrícia Gonçalves Saldanha, Marcelo Rodrigo de Avelar Bastos Alves

DADOS ABERTOS À BRASILEIRA: ASPECTOS DE UMA CIDADANIA DENEGADA

Claudomilson Fernandes Braga, Simone Antoniaci Tuzzo

MÍdIAS SOCIAIS E RADIODIFUSÃo COMUNITÁRIA COMO PLATAFORMAS COMPLEMENTARES

Adilson Vaz Cabral Filho, Cinthya Pires Oliveira

COMUNICAÇÃo de INTERESSE PÚBLICO E CIBERATIVISMO: AS AÇÕES DE SUSTENTABILIDADE DA COMUNIDADE VIRTUAL MAPA DO LIXO BELÉM (PA) - AMAZÔNIA

Neusa Pressler, Vanda do Socorro Furtado Amin

A Transparência Administrativa e Comunicação Pública: ANÁLISE DO CASO "FUNCIONÁRIOS-FANTASMAS" DA ASSEMBLEIA Legislativa do Maranhão

Asmynne Bárbara Barbosa dos Santos, José Ferreira Júnior

AÇões do FNDC NO DEBATE SOBRE A DEMOCRATIZAÇÃo DA COMUNICAÇÃO: BREVES APONTAMENTOS

Carlos Henrique Demarchi, Maria Teresa Miceli Kerbauy

A minha família eXISTe? DeliberaÇão e CONVERSaÇão POLítica NA INTERNET

Danila Gentil Rodriguez Cal, Elias Santos Serejo 
